\section{Stevastelin A3, D3 and E3, Novel Congeners from a High Producing Mutant of Penicillium sp.}

\author{
Tomio Morino, Kei-IChi Shimada, Akira Masuda, \\ Masakazu Nishimoto and SeIIChi Saito
}

Research and Development Division, Pharmaceuticals Group, Nippon Kayaku Co., Ltd., 31-12, 3-chome, Shimo, Kita-ku, Tokyo 115, Japan

(Received for publication May 17, 1996)

In our previous studies, four stevastelin congeners named A, B, B3 and C3 have been isolated from culture broth of a fungus, Penicillium, as immunosuppressants ${ }^{1)}$. Structures of these stevastelins have been elucidated to be cyclic depsipeptides ${ }^{2,3}$. In the course of ultraviolet treatment of the strain, we found that a high producing mutant yielded three novel stevastelin congeners. In this report we describe isolation, physico-chemical properties, structural elucidation and immunosuppressive activities of these novel stevastelin congeners named A3, D3 and E3.

In order to isolate a high producing mutant, spores of the stevastelin producing strain, Penicillium sp. NK $374186^{1}$, were irradiated with a UV lamp under a condition giving $1 \%$ survival rate. About a hundred survival strains were picked and cultured as described previously ${ }^{11}$. From the mycelium of each culture, stevastelins were extracted with $\mathrm{MeOH}$, and again extracted with EtOAc after removal of the initial solvent. These extracts were applied to TLC analysis developed with a mixture of $\mathrm{CHCl}_{3}-\mathrm{MeOH}, 20: 1(\mathrm{v} / \mathrm{v})$, and stevastelins were detected with toluidine-chlorine color reaction. With this screening, a strain which produced the known stevastelins at levels about five times as much as the original strain was picked. Furthermore, this strain gave additional positive spots at $\mathrm{Rf}$ values of $0.06,0.07$ and 0.42 , whereas $R f$ values of the known stevastelins $\mathrm{A}, \mathrm{B}, \mathrm{B} 3$ and $\mathrm{C} 3$ are $0.12,0.35,0.49$ and 0.33 , respectively ${ }^{1)}$. Tentatively, the compounds corresponding to these unidentified spots were named stevastelin A3 $(\mathrm{Rf}=0.07), \mathrm{D} 3(\mathrm{Rf}=0.06)$ and $\mathrm{E} 3(\mathrm{Rf}=0.42)$, respectively.

The high producing mutant was cultured in a 200 liter jar fermenter as described for the original strain ${ }^{1}$. From mycelia collected from combined 600 liters of the culture broth, the stevastelins were extracted with 300 liters of $\mathrm{MeOH}$. The $\mathrm{MeOH}$ extract was diluted with 300 liters of water and applied to a Diaion HP-20 resin (20 liters). After washing with $50 \%$ aqueous $\mathrm{MeOH}$, stevastelin A3 and D3 were eluted with 30 liters of $60 \%$ aqueous $\mathrm{Me}_{2} \mathrm{CO}$, and stevastelin E3 was eluted with 30 liters of $80 \%$ aqueous $\mathrm{Me}_{2} \mathrm{CO}$, stepwisely. The mixture of stevastelin A3 and D3 were applied to Diaion CHP20P resin (2 liters) and eluted with a linear gradient from $50 \%$ to $70 \%$ aqueous $\mathrm{Me}_{2} \mathrm{CO}$ in order to separate stevastelin A3 and D3. These stevastelins were further purified by Sephadex LH-20 column chromatogaraphy $\left(\mathrm{CHCl}_{3}-\mathrm{MeOH}=1: 1\right)$, respectively, to give $800 \mathrm{mg}$ of stevastelin A3 and $130 \mathrm{mg}$ of stevastelin D3 as a colorless powder. On the other hand stevastelin $\mathrm{E} 3$ was purified by two successive silica gel $\left(\mathrm{CHCl}_{3}-\mathrm{MeOH}=50: 1\right.$ and $n$-hexane $\left.-\mathrm{Me}_{2} \mathrm{CO}=3: 1\right)$ and Sephadex LH-20 $\left(\mathrm{CHCl}_{3}\right.$ $\mathrm{MeOH}=1: 1$ ) column chromatography to give $500 \mathrm{mg}$ as a colorless powder.

Stevastelin A3, D3 and E3 are soluble in $\mathrm{CHCl}_{3}$, EtOAc, and MeOH. Spectroscopic data of FAB-MS and NMR analysis and other physico-chemical properties of stevastelin A3, D3 and E3 are shown in Table 1. These data of stevastelin A3, D3 and E3 are very similar to that of stevastelin $\mathrm{B}^{2)}$ (Table 1), confirming that these compounds are stevastelin congeners.

Structure of stevastelin B3 was reported to be composed of four moieties, $O$-acetyl-L-serine, L-threonine, L-valine and 3,5-dihydroxy-2,4-dimethylstearic acid ${ }^{2,3}$ ) (Fig. 1, Table 1). NMR data of stevastelin A3 is similar to stevastelin $\mathrm{B} 3$ with regard to $O$-acetylserine, valine and the fatty acid moieties (Table 1), indicating the presence of these moieties. However, the T3 carbon of stevastelin A3 is shifted downfield $\left(\delta_{\mathrm{C}} 70.60\right.$, Table 1$)$ when compared with that of stevastelin $\mathrm{B} 3\left(\delta_{\mathrm{C}} 65.13\right.$, Table 1), and the signal due to the T3 hydroxy group is not detected (Table 1). These results suggest a substitution of the T3 hydroxy group of stevastelin A3. Molecular formulas of stevastelin A3 and B3 show that stevastelin $\mathrm{A} 3$ has one more sulfur atom and three more oxygen atoms than stevastelin B3 (Table 1). Based on these data, the T3 hydroxy group of stevastelin A3 is

Fig. 1. Structures of stevastelin A3, B3, D3 and E3.
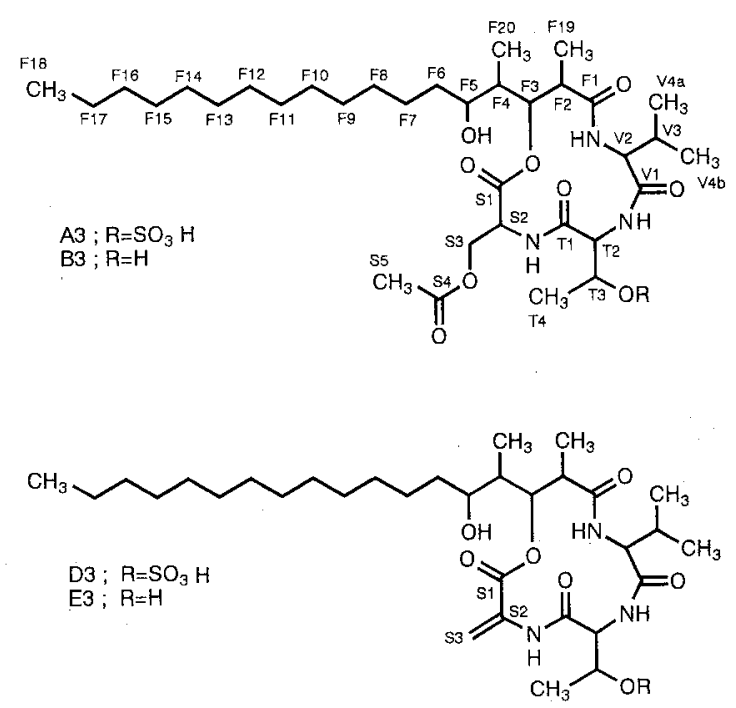
Table 1. Physico-chemical properties of stevastelins.

\begin{tabular}{|c|c|c|c|c|c|c|c|c|}
\hline \multirow{2}{*}{$\begin{array}{l}\text { NMR data } \\
\text { Carbon No. }\end{array}$} & \multicolumn{2}{|r|}{$B 3^{c}$} & \multicolumn{2}{|r|}{$\mathrm{A} 3$} & \multicolumn{2}{|r|}{ D3 } & \multicolumn{2}{|r|}{ E3 } \\
\hline & C ppm & $\begin{array}{c}\mathrm{H} \mathrm{ppm} \mathrm{mH} \\
(J=(\mathrm{Hz}))\end{array}$ & C ppm & $\begin{array}{c}\mathrm{H} \text { ppm mH } \\
(J=(\mathrm{Hz}))\end{array}$ & C ppm & $\begin{array}{c}\mathrm{H} \text { ppm mH } \\
(J=(\mathrm{Hz}))\end{array}$ & C ppm & $\begin{array}{c}\text { H ppm mH } \\
(J=(\mathrm{Hz}))\end{array}$ \\
\hline \multicolumn{9}{|l|}{ [Fatty acid] } \\
\hline $\mathrm{F} 1$ & $170.43 \mathrm{~s}$ & - & $170.74^{\mathrm{d}} \mathrm{s}$ & - & $171.23^{\mathrm{e}} \mathrm{s}$ & - & $171.45^{\mathrm{r}} \mathrm{s}$ & - \\
\hline $\mathrm{F} 2$ & $41.01 \mathrm{~d}$ & $2.90 \mathrm{~m}$ & $41.67 \mathrm{~d}$ & $2.87 \mathrm{~m}$ & $40.79 \mathrm{~d}$ & $2.99 \mathrm{~m}$ & $40.60 \mathrm{~d}$ & $3.01 \mathrm{~m}$ \\
\hline $\mathrm{F}^{3}$ & $80.13 \mathrm{~d}$ & $4.91 \mathrm{~m}$ & $79.62 \mathrm{~d}$ & $4.90 \mathrm{~m}$ & $81.19 \mathrm{~d}$ & $4.99 \mathrm{~m}$ & $81.59 \mathrm{~d}$ & $5.00 \mathrm{~m}$ \\
\hline F4 & $38.78 \mathrm{~d}$ & $1.73 \mathrm{~m}$ & $39.10 \mathrm{~d}$ & $1.62 \mathrm{~m}$ & $39.20 \mathrm{~d}$ & $1.90 \mathrm{~m}$ & $39.20 \mathrm{~d}$ & $1.96 \mathrm{~m}$ \\
\hline F5 & $68.85 \mathrm{~d}$ & $\begin{array}{l}3.84 \mathrm{br} \\
-\mathrm{OH}, 4.37 \mathrm{~d}(5.8)\end{array}$ & $68.70 \mathrm{~d}$ & $\begin{array}{l}3.84 \mathrm{br} \\
-\mathrm{OH}, 4.32 \mathrm{~m}\end{array}$ & $68.85 \mathrm{~d}$ & $\begin{array}{l}3.85 \mathrm{br} \\
-\mathrm{OH}, 4.44 \mathrm{~m}\end{array}$ & $68.86 \mathrm{~d}$ & $\begin{array}{l}3.86 \mathrm{br} \\
-\mathrm{OH}, 4.45 \mathrm{~m}\end{array}$ \\
\hline F6 & $34.79 \mathrm{t}$ & $\begin{array}{l}1.20 \mathrm{~m} \\
1.34 \mathrm{~m}\end{array}$ & $34.81 \mathrm{t}$ & $\begin{array}{l}1.20 \mathrm{~m} \\
1.32 \mathrm{~m}\end{array}$ & $34.84 \mathrm{t}$ & $1.362 \mathrm{H} \mathrm{br}$ & $34.84 \mathrm{t}$ & $1.362 \mathrm{H} \mathrm{br}$ \\
\hline F7 & $25.62 \mathrm{t}$ & $1.242 \mathrm{H}$ & $25.63 \mathrm{t}$ & $1.242 \mathrm{H}$ & $25.60 \mathrm{t}$ & $1.242 \mathrm{H}$ & $25.59 \mathrm{t}$ & $1.242 \mathrm{H}$ \\
\hline F8 & 28.6 & $1.2416 \mathrm{H}$ & 28.6 & $1.2416 \mathrm{H}$ & 28.6 & $1.24 \mathrm{I} 6 \mathrm{H}$ & 28.6 & $1.2416 \mathrm{H}$ \\
\hline$\sim 15$ & $\sim 29.0 \mathrm{t} \times 8$ & & $\sim 29.0 \mathrm{t} \times 8$ & & $\sim 29.0 \mathrm{t} \times 8$ & & $\sim 29.0 \times 8$ & \\
\hline F16 & $31.21 \mathrm{t}$ & $1.242 \mathrm{H}$ & $31.21 \mathrm{t}$ & $1.242 \mathrm{H}$ & $31.21 \mathrm{t}$ & $1.242 \mathrm{H}$ & $31.21 \mathrm{t}$ & $1.242 \mathrm{H}$ \\
\hline F17 & $22.01 \mathrm{t}$ & $1.242 \mathrm{H}$ & $22.00 \mathrm{t}$ & $1.242 \mathrm{H}$ & $22.00 \mathrm{t}$ & $1.242 \mathrm{H}$ & $22.00 \mathrm{t}$ & $1.242 \mathrm{H}$ \\
\hline F18 & $13.86 \mathrm{q}$ & $0.86+3 \mathrm{H}(6.8)$ & $13.85 \mathrm{q}$ & $0.85 \mathrm{t} 3 \mathrm{H}(6.7)$ & $14.00 \mathrm{q}$ & $0.84 \mathrm{t} 3 \mathrm{H}(6.9)$ & $14.01 \mathrm{q}$ & $0.85+3 \mathrm{H}(7.0)$ \\
\hline F19 & $13.72 \mathrm{q}$ & $1.12 \mathrm{~d} 3 \mathrm{H}(7.3)$ & $13.57 \mathrm{q}$ & $1.11 \mathrm{~d} 3 \mathrm{H}(7.0)$ & $13.87 \mathrm{q}$ & $1.17 \mathrm{~d} 3 \mathrm{H}(7.0)$ & $13.85 \mathrm{q}$ & $1.17 \mathrm{~d} 3 \mathrm{H}(6.2)$ \\
\hline $\mathrm{F} 20$ & $9.11 \mathrm{q}$ & $0.54 \mathrm{~d} 3 \mathrm{H}(6.6)$ & $9.03 \mathrm{q}$ & $0.52 \mathrm{~d} 3 \mathrm{H}(7.0)$ & $9.15 \mathrm{q}$ & $0.55 \mathrm{~d} 3 \mathrm{H}(6.6)$ & $9.15 \mathrm{q}$ & $0.56 \mathrm{~d} 3 \mathrm{H}(6.6)$ \\
\hline \multicolumn{9}{|l|}{ [Valine] } \\
\hline V1 & $170.76 \mathrm{~s}$ & - & $170.02^{d} \mathrm{~s}$ & - & $170.08^{\mathrm{e}} \mathrm{s}$ & _- & $169.98^{\mathrm{f}} \mathrm{s}$ & - \\
\hline $\mathrm{V} 2$ & $61.51 \mathrm{~d}$ & $\begin{array}{l}4.03 \mathrm{~m} \\
-\mathrm{NH}, 7.67 \mathrm{br}\end{array}$ & $61.13 \mathrm{~d}$ & $\begin{array}{l}3.98 \mathrm{~m} \\
-\mathrm{NH}, 7.28 \mathrm{~d}(10.3)\end{array}$ & $61.23 \mathrm{~d}$ & $\begin{array}{l}4.14 \mathrm{~m} \\
-\mathrm{NH}, 7.97 \mathrm{~d}(9.9)\end{array}$ & $61.33 \mathrm{~d}$ & $\begin{array}{l}4.15 \mathrm{~m} \\
-\mathrm{NH}, 7.90 \mathrm{~d}(9.2)\end{array}$ \\
\hline v3 & $28.63 \mathrm{~d}$ & $2.05 \mathrm{~m}$ & $27.88 \mathrm{~d}$ & $2.03 \mathrm{~m}$ & $28.60 \mathrm{~d}$ & $2.04 \mathrm{~m}$ & $29.11 \mathrm{~d}$ & $2.05 \mathrm{~m}$ \\
\hline $\mathrm{V} 4 \mathrm{a}$ & $18.87 \mathrm{q}$ & $0.90 \mathrm{~d} 3 \mathrm{H}(6.6)$ & $18.32 \mathrm{q}$ & $0.85 \mathrm{~d} 3 \mathrm{H}(6.6)$ & $18.13 \mathrm{q}$ & $0.88 \mathrm{~d} 3 \mathrm{H}(6.6)$ & $19.12 \mathrm{q}$ & $0.89 \mathrm{~d} 3 \mathrm{H}(6.2)$ \\
\hline $\mathrm{V} 4 \mathrm{~b}$ & $19.11 \mathrm{q}$ & $0.94 \mathrm{~d} 3 \mathrm{H}(6.6)$ & $18.83 \mathrm{q}$ & $0.90 \mathrm{~d} 3 \mathrm{H}(6.6)$ & $19.03 q$ & $0.93 \mathrm{~d} 3 \mathrm{H}(6.6)$ & $20.51 \mathrm{q}$ & $0.94 \mathrm{~d} 3 \mathrm{H}(6.6)$ \\
\hline \multicolumn{9}{|l|}{ [Threonine] } \\
\hline $\mathrm{T} 1$ & $170.58 \mathrm{~s}$ & - & $169.42^{\mathrm{d}} \mathrm{s}$ & - & $167.35^{\mathrm{c}} \mathrm{s}$ & - & $168.84^{E} \mathrm{~s}$ & - \\
\hline $\mathrm{T} 2$ & $59.30 \mathrm{~d}$ & $\begin{array}{l}4.16 \mathrm{~m} \\
-\mathrm{NH}, 7.72 \mathrm{~d}(9.9)\end{array}$ & $60.51 \mathrm{~d}$ & $\begin{array}{l}3.94 \mathrm{~m} \\
-\mathrm{NH}, 8.23 \mathrm{~d}(9.5)\end{array}$ & $57.29 \mathrm{~d}$ & $\begin{array}{l}4.35 \mathrm{~m} \\
-\mathrm{NH}, 8.15 \mathrm{~d}(9.2)\end{array}$ & $57.48 \mathrm{~d}$ & $\begin{array}{l}4.31 \mathrm{~d}(9.5) \\
-\mathrm{NH}, 8.25 \mathrm{~d}(9.5)\end{array}$ \\
\hline $\mathrm{T} 3$ & $65.13 \mathrm{~d}$ & $\begin{array}{l}4.03 \mathrm{~m} \\
-\mathrm{OH}, 5.24 \mathrm{~d}(4.4)\end{array}$ & $70.60 \mathrm{~d}$ & $4.32 \mathrm{~m}$ & $70.58 \mathrm{~d}$ & $4.88 \mathrm{~m}$ & $65.26 \mathrm{~d}$ & $\begin{array}{l}4.50 \mathrm{~m} \\
-\mathrm{OH}, 5.28 \mathrm{~d}(4.0)\end{array}$ \\
\hline $\mathrm{T} 4$ & $20.84 \mathrm{q}$ & $1.08 \mathrm{~d} 3 \mathrm{H}(6.2)$ & $19.25 \mathrm{q}$ & $1.17 \mathrm{~d} 3 \mathrm{H}(6.2)$ & $19.16 \mathrm{q}$ & $1.21 \mathrm{~d} 3 \mathrm{H}(6.2)$ & $19.12 \mathrm{q}$ & $1.04 \mathrm{~d} 3 \mathrm{H}(6.2)$ \\
\hline \multicolumn{9}{|c|}{$[O$-Acetylserine $]$} \\
\hline $\mathrm{S} 1$ & $168.72 \mathrm{~s}$ & -_ & $168.85^{\mathrm{d}} \mathrm{s}$ & - & $162.09^{\mathrm{e}} \mathrm{s}$ & - & $161.67^{\circ} \mathrm{s}$ & - \\
\hline S2 & $50.06 \mathrm{~d}$ & $\begin{array}{l}4.91 \mathrm{~m} \\
-\mathrm{NH}, 7.49 \mathrm{~d}(8.8)\end{array}$ & $49.35 \mathrm{~d}$ & $\begin{array}{l}4.92 \mathrm{~m} \\
-\mathrm{NH}, 7.94 \mathrm{~d}(7.0)\end{array}$ & $131.53 \mathrm{~s}$ & $-\overline{\mathrm{NH}}, 8.30 \mathrm{~s}$ & $131.58 \mathrm{~s}$ & $-\overline{\mathrm{NH}}, 8.29 \mathrm{~s}$ \\
\hline S3 & $63.15 \mathrm{t}$ & $\begin{array}{l}4.20 \mathrm{~m} \\
4.35 \mathrm{~m}\end{array}$ & $63.21 \mathrm{t}$ & $\begin{array}{l}4.25 \mathrm{~m} \\
4.33 \mathrm{~m}\end{array}$ & $109.76 \mathrm{t}$ & $\begin{array}{l}5.77 \mathrm{~s} \\
6.27 \mathrm{~s}\end{array}$ & $107.96 \mathrm{t}$ & $\begin{array}{l}5.74 \mathrm{~s} \\
6.39 \mathrm{~s}\end{array}$ \\
\hline S4 & $170.04 \mathrm{~s}$ & - & $169.82^{\mathrm{d}} \mathrm{s}$ & - & - & - & - & - \\
\hline S5 & $20.55 \mathrm{q}$ & $2.01 \mathrm{~s} 3 \mathrm{H}$ & $20.45 \mathrm{q}$ & $2.00 \mathrm{~s} 3 \mathrm{H}$ & - & - & - & - \\
\hline \multicolumn{9}{|l|}{ FAB-MS $m / z$} \\
\hline Found & \multicolumn{2}{|c|}{$678(\mathrm{M}+\mathrm{Na})^{+}$} & \multicolumn{2}{|c|}{$\begin{array}{l}734(\mathrm{M}-\mathrm{H})^{-} \\
780(\mathrm{M}+2 \mathrm{Na}-\mathrm{H})^{+}\end{array}$} & \multicolumn{2}{|c|}{$\begin{array}{l}674(\mathrm{M}-\mathrm{H})^{-} \\
720(\mathrm{M}+2 \mathrm{Na}-\mathrm{H})^{+}\end{array}$} & \multicolumn{2}{|c|}{$594(\mathrm{M}-\mathrm{H})^{-}$} \\
\hline $\begin{array}{l}\text { mol } \\
\text { formula } \\
\text { mol weight } \\
\text { UV } \lambda_{\text {max }}^{\mathrm{MeOH}}\end{array}$ & \multicolumn{2}{|c|}{$\begin{array}{ll} & \mathrm{C}_{34} \mathrm{H}_{61} \mathrm{~N}_{3} \mathrm{O}_{9} \\
\mathrm{t} & 655.85\end{array}$} & \multicolumn{2}{|c|}{$\begin{array}{l}\mathrm{C}_{34} \mathrm{H}_{61} \mathrm{~N}_{3} \mathrm{O}_{12} \mathrm{~S} \\
735.91\end{array}$} & $\begin{array}{l}\mathrm{C}_{32} \mathrm{H}_{57} \mathrm{~N} \\
675.86\end{array}$ & $\mathrm{O}_{10} \mathrm{~S}$ & $\begin{array}{l}\mathrm{C}_{32} \mathrm{H}_{57} \mathrm{~N} \\
595.79\end{array}$ & ${ }_{3} \mathrm{O}_{7}$ \\
\hline$(\mathrm{E} 1 \%)$ & - & & - & & $242 \mathrm{~nm}(9$ & $6.64)$ & $242 \mathrm{~nm}($ & $05.21)$ \\
\hline
\end{tabular}

a Recorded in DMSO- $d_{6}$.

b Refered to Fig. 1.

c Cited from ref. 2.

$\mathrm{d} \sim \mathrm{f}$ These assignments are exchangable.

concluded to be $O$-sulfonylated (Fig. 1).

NMR data of stevastelin D3 are similar to that of stevastelin B3 with regard to valine and the fatty acid moieties (Table 1). The data of the threonine moiety of stevastelin D3 is also similar to that of stevastelin A3 (Table 1). These data indicate the presence of valine, the fatty acid and $O$-sulfonylthreonine moieties in stevastelin D3 (Fig. 1). However, in the ${ }^{1} \mathrm{H}$ NMR spectrum of stevastelin D3, signals due to the acetyl group of the $O$-acetylserine moiety are not detected (S4,5, Table 1$)$ and signals due to olefinic protons $\left(\delta_{\mathrm{H}} 5.77[\mathrm{~s}], 6.27[\mathrm{~s}]\right.$,
Table 1) are newly detected. A presence of olefinic protons is supported by ${ }^{13} \mathrm{C}$ NMR spectrum detected at $\delta_{\mathrm{C}} 109.76[\mathrm{t}]$ and $\delta_{\mathrm{C}} 131.53$ [s] (S3 and S2, Table 1). Also a singlet amide proton $\left(\delta_{\mathrm{H}} 8.29[\mathrm{~s}]\right.$, Table 1$)$ and carbonyl carbon $\left(\delta_{\mathrm{c}} 162.09\right.$ [s], S1, Table 1) are detected in the spectrum of stevastelin D3. From these data stevastelin D3 is concluded to have a dehydroalanine moiety, $-\mathrm{NH}-\stackrel{\mathrm{II}}{\mathrm{C}}-\mathrm{CO}-$, instead of the $O$-acetylserine moiety (Fig. 1).

NMR data of stevastelin E3 are similar to stevastelin 
Table 2. Effect of stevastelin congeners on human $\mathrm{T}$ lymphocyte blastogenesis ${ }^{\mathrm{a}}$.

\begin{tabular}{cc}
\hline Compound & $\mathrm{IC}_{50}(\mu \mathrm{g} / \mathrm{ml})$ \\
\hline B3 & $0.42^{\mathbf{b}}$ \\
A3 & 30.0 \\
D3 & 28.0 \\
E3 & 1.8 \\
\hline
\end{tabular}

a Human $\mathrm{T}$ lymphocytes were isolated from peripheral blood by density gradient centrifugation. Cells were collected and washed twice with PBS and inoculated into 96-well microplate with $10 \%$ FCS-RPMI1640 medium containing $10 \mathrm{~mm}$ HEPES buffer. 1 hour after sample addition, OKT-3 antibody was added at a final concentration of $2 \mathrm{ng} / \mathrm{ml}$. T cell growth was monitored at 3 days with ${ }^{3} \mathrm{H}$-thymidine uptake activity.

b Cited from ref. 1.

B3 regarding the fatty acid, valine and threonine moieties (Table 1), and are also similar to stevastelin D3 regarding the dehydroalanine moiety (Table 1). These results indicate the structure of stevastelin E3 with these moieties as presented (Fig. 1).

Cyclic structures of the stevastelins were reporterd to be formed by ester linkages between a carboxylic group of the $O$-acetylserine and the F5-hydroxy group in stevastelin $\mathrm{B}^{2)}$, and the F3-hydroxy group in stevastelin B3 (Fig. 1) ${ }^{2}$. Chemical shifts of the F3 carbons of stevastelin A3, D3 and E3 $\left(\delta_{\mathrm{C}} 79.62,81.19,81.59\right.$, respectively, Table 1) are lower than those of the F5 carbons $\left(\delta_{\mathrm{C}} 68.70,68.85,68.86\right.$, respectively, Table 1$)$ : Furthermore, the F3 hydroxy groups of the fatty acid moieties were not detected in the NMR spectra of stevastelin A3, D3 and E3, whereas the F5 hydroxy groups were detected (Table 1). These results are quite similar to stevastelin B3 (Table 1), and show that ester linkages are formed with the F3 hydroxy groups (Fig.1) as described previously ${ }^{2}$.

Immunosuppressive activities of stevastelin A3, D3 and $\mathrm{E} 3$ against $\mathrm{OKT}-3$ stimulated human $\mathrm{T}$ cell proliferation are shown in Table 2. Stevastelin A3 and D3 show weak activities, whereas stevastelin E3 shows potent activity. This result suggests that the $O$-sulfonylation of the T3 hydroxy group drastically weaken immunosuppressive activity against $\mathrm{T}$ cell blastogenesis. On the other hand, an alteration of the $O$-acetylserine moiety to the dehydroalanine moiety seems to be less influential on the immunosuppressive activity of stevastelin (Table 2).

In this report novel three stevastelin congeners, A3, D3 and E3, were isolated from a cultured broth of the high producing mutant, based on the color reaction to toluidine-chlorine reagent. A congener, stevastelin E3, showed comparable immunosuppressive activity to the known stevastelin congener ${ }^{1)}$. Stevastelin A3 and D3 showed weak activities; however, these compounds were useful to investigate the structure-activity relationship of stevastelin. Another stevastelin congeners with modified amino acid moieties are constructed by chemical conversions (unpublished result). Precise structure-activity relationship of stevastelin will be examined with these congeners and will be reported elsewhere.

\section{Acknowledgments}

We thank Mr. Masaya Sato and Dr. Yutaka Hashimoto for their technical assistance and valuable discussion on the spectroscopic study. We also thank Mrs. ERIKo TANAKATOYADA for her excellent technical assistance on the assay.

\section{References}

1) Morino, T.; A. Masuda, M. Yamada, M. Nishimoto, T. Nishikiori, S. SAIto \& N. Shimada: Stevastelins, novel immunosuppressants produced by Penicillium. J. Antibiotics 47: $1341 \sim 1343,1994$

2) Morino, T.; K. Shimada, A. Masuda, N. Yamashita, M. Nishimoto, T. Nishikiori \& S. SAito: Structural determination of stevastelins, novel depsipeptides from Penicillium sp. J. Antibiotics 49: 564 568, 1996

3) Shimada, K.; T. Morino, A. Masuda, M. Sato, M. KitaGawa \& S. SAITo: Absolute structural determination of stevastelin B. J. Antibiotics 49: 569 574, 1996 